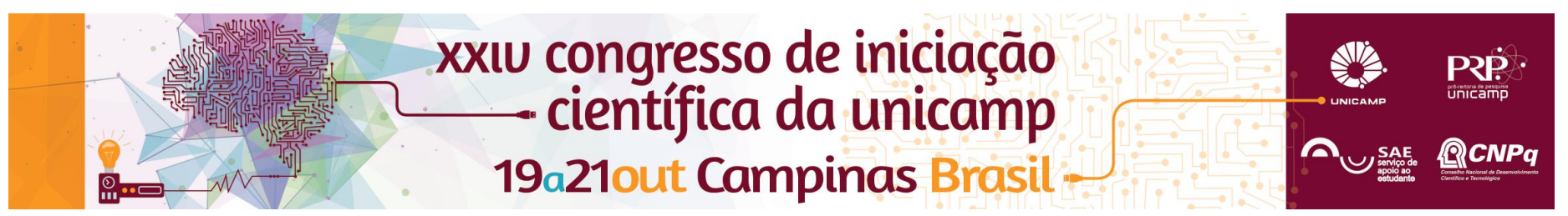

\title{
Subjective evaluation of contrast resolution on different digital imaging systems
}

\author{
Sabrina C. Prado*, Carlos A. S. Lima, Deborah Q. Freitas, Francisco Haiter-Neto, Matheus L Oliveira.
}

\begin{abstract}
Since contrast resolution of current radiographic systems exceed human visual perception, the aim of this study was to subjectively evaluate the contrast resolution of different digital radiographic systems. Five radiographic phantoms were prepared containing a solution of dipotassium phosphate (K2HPO4) in 6 different volumes. Each phantom presented the solutions of K2HPO4 in a different sequence and was X-rayed with 5 digital radiographic systems: Digora Toto, Digora Optime, Snapshot, Express and VistaScan. All 25 digital radiographic images were exported, stored in TIFF format and evaluated in the JPEGView software. Ten examiners had to identify the sequence between the lowest and the highest volume of K2HPO4 solution. For each image, the percentage of correct answers was calculated. After 15 days, all images were re-evaluated to test the intra-rater reproducibility. After exploratory analysis of the data in the IBM SPSS software, it was found that the data were not normally distributed using the Shapiro-Wilk test $(p>0.05)$. Thus, the Mann-Whitney test compared the digital systems in pairs, with a significance level of $5 \%(\alpha=0.05)$. Intraclass correlation analysis assessed the level of intra-examiner agreement. Intraclass correlation coefficient (ICC) ranged from moderate to almost perfect. The Digora Toto and Express were the systems with the highest values of percentage of correct answers. The Snapshot presented intermediate values and did not differ from Express and Digora Optime. The Digora Optime and VistaScan were the systems with the lowest values of percentage of correct answers. In conclusion, among the digital radiographic systems evaluated, Digora Toto and Express produced radiographic images with the best subjective contrast resolution.
\end{abstract}

Key words: Radiographic image enhancement, Contrast sensitivity, Technology Assessment, Biomedical.

\section{Introduction}

Despite the possibility of detecting subtle differences in radiographic contrast with digital objective analysis ${ }^{1,2}$, contrast resolution of current radiographic systems exceed human visual perception.

The aim of this study was to subjectively evaluate the contrast resolution of different digital radiographic systems.

\section{Results and Discussion}

The Digora Toto and Express did not differ significantly from each other $(p>0.05)$ and were the systems with the highest values of percentage of correct answers. The Express system did not differ significantly from the Snapshot, which did not differ from Digora Optime $(p>0.05)$. The Digora Optime and VistaScan did not differ significantly from each other different $(p>0.05)$ and were the systems with the lowest values of percentage of correct answers. Any other comparison differed significantly $(p<0.05$, Table 1$)$.

Intraclass correlation coefficient (ICC) ranged from moderate to almost perfect. Two examiners were excluded for obtaining insignificant ICC values (Table 2).

Table 1: Mean values and standard deviation $( \pm S D)$ of percentage of correct answers of digital radiographic systems.

\begin{tabular}{cccc}
\hline Technology & System & Mean & SD \\
\hline Sensor & Digora Toto & $96.39 \mathrm{a}$ & 2.89 \\
PSP & Express & $95.00 \mathrm{ab}$ & 1.97 \\
Sensor & Snapshot & $92.78 \mathrm{bc}$ & 3.09 \\
PSP & Digora Optime & $87.78 \mathrm{~cd}$ & 3.94 \\
PSP & VistaScan & $84.42 \mathrm{~d}$ & 7.00 \\
\hline
\end{tabular}

Table 2: Mean values ( \pm standard deviation) of the examiners in both evaluations, Intraclass correlation

\begin{tabular}{ccccc}
\multicolumn{2}{c}{ coefficient } & $(\mathrm{ICC})$ & \multicolumn{2}{l}{ and } \\
\hline Examiner & Evaluation & Re-evaluation & ICC & Cl95\% \\
\hline 1 & $90.67(2.90)$ & $90.67(0.99)$ & 0.643 & $-2.43-0.96$ \\
2 & $94.67(4.61)$ & $89.78(4.33)$ & 0.883 & $-0.12-0.98$ \\
3 & $92.44(5.58)$ & $93.78(4.55)$ & 0.743 & $-1.47-0.97$ \\
4 & $88.89(6.09)$ & $89.78(8.55)$ & 0.865 & $-0.29-0.98$ \\
5 & $92.00(4.87)$ & $94.67(2.98)$ & 0.852 & $-0.42-0.98$ \\
6 & $90.67(3.98)$ & $90.22(4.33)$ & 0.986 & $0.86-0.99$ \\
7 & $92.89(5.53)$ & $92.00(3.72)$ & 0.953 & $0.55-0.99$ \\
8 & $82.53(16.69)$ & $81.11(14.99)$ & 0.658 & $-2.28-0.96$ \\
\hline
\end{tabular}

\section{Conclusions}

Among the digital radiographic systems evaluated, Digora Toto and Express produced radiographic images with the better subjective contrast resolution.

\section{Acknowledgement}

We thank CNPq/PIBIC-EM for supporting this research and granting the scholarships.

\footnotetext{
${ }^{1}$ Shah, N.; Bansal, N.; Logani, A. Recent advances in imaging technologies in dentistry. World J Radiol. 2014, 6,:794-807.

${ }^{2}$ Brüllmann, D.D.; Kempkes, B.; d'Hoedt, B.; Schulze, R. Contrast curves of five different intraoral X-ray sensors: a technical note. Oral Surg Oral Med Oral Pathol Oral Radiol. 2013, 115:e55-61.
} 